\title{
Detection and Identification of PQ Disturbances Using S-Transform and Artificial Intelligent Technique
}

\author{
Ahmed Hussain Elmetwaly ${ }^{1}$, Abdelazeem Abdallah Abdelsalam ${ }^{2, ~ *, ~ A z z a ~ A h m e d ~ E l d e s s o u k y ~}{ }^{3}$, \\ Abdelhay Ahmed Sallam ${ }^{3}$
}

${ }^{1}$ Dept. of Electrical Engineering, El Shorouk Academy, Cairo, Egypt

${ }^{2}$ Dept.of Electrical Engineering, Suez Canal University, Ismailia, Egypt

${ }^{3}$ Dept. of Electrical Engineering, Port-Said University, Port-Said, Egypt

\section{Email address:}

eng.ahmedhussain7@gmail.com (A. H. Elmetwaly), aaabdelsalam@eng.suez.edu.eg (A. A. Abdelsalam), azzaeldessouky@yahoo.com (A. A. Eldessouky), aasallam@ucalgary.ca (A. A. Sallam)

\section{To cite this article:}

Ahmed Hussain Elmetwaly, Abdelazeem Abdallah Abdelsalam, Azza Ahmed Eldessouky, Abdelhay Ahmed Sallam. Detection and Identification of PQ Disturbances Using S-Transform and Artificial Intelligent Technique. American Journal of Electrical Power and Energy Systems. Vol. 4, No. 1, 2015, pp. 1-9. doi: 10.11648/j.epes.20150401.11

\begin{abstract}
This paper proposes a new technique based on S-transform time-frequency analysis and Fuzzy expert system for classifying power quality (PQ) disturbances. The S-transform is a new time frequency analysis method. It has the features of both continuous wavelet transform (CWT) and short time Fourier transform (STFT). Through S-transform time-frequency analysis, a set of feature components are extracted for identifying PQ disturbances such as; the amplitude of the S-transform matrix and the total harmonic distortion (THD). The two parameters are the inputs to Fuzzy-expert system that uses some rules on these inputs to characterize the PQ events in the captured waveform (e.g. sag, swell, interruption, surge, sag with harmonic and swell with harmonic). Several simulation using Matlab environment and practical data are used to validate the proposed technique. The results depict that the proposed technique has the ability to accurately identify and characterize PQ disturbances.
\end{abstract}

Keywords: Power Quality, S-Transform, Fuzzy Expert System

\section{Introduction}

In a power system; switching, faults, dynamics, or nonlinear loads cause various types of power quality (PQ) disturbances such as surges, harmonics, interruptions, sags, swells, etc. [1]. In order to improve the quality of power supply, it is necessary to make clear the sources and causes of PQ disturbances before appropriate mitigating actions can be taken. To analyze PQ disturbances, short time discrete Fourier transform (STFT) [2, 3] is mostly often used. This transform has been successfully used for stationary signals where properties of signals do not evolve in time. For nonstationary signals, the STFT does not track the signal dynamics properly due to the limitations of a fixed window width chosen in advance. Thus, STFT cannot be successfully used to analyze transient signals comprising both high and low-frequency components.

On the other hand, wavelet transform [4- 6] is a notable tool for detection, localization and classification of the disturbances. However, the noises will lower down the performance of wavelet as the spectrum of noises overlaps with that of power quality disturbances.

Kalman filter can be employed to detect and to analyze voltage event [7, 8]. The results of Kalman filter depend on the model of the system used as well as the appropriate selection of the filter parameters is not guaranteed, the rate of convergence of the results will be slow or the results will diverge.

The S-transform (ST) [9-11] is an extension of continuous wavelet transforms (CWT) and STFT. Because of its good time-frequency characteristic, it is very adequate for PQ disturbances analysis and classification. The classification of PQ disturbances can be done by applying artificial intelligent techniques like artificial neural network [12], Fuzzy logic [13], and support vector machine (SVM) [14].

This paper proposes a Fuzzy expert system for making a decision based on the features extracted from S-transform. These features are the amplitude of the captured waveform and the total harmonic distortion. The tested waveforms are generated using Matlab environment software and also an IEEE practical data. 


\section{The Proposed Classification Methodology}

The block diagram of the proposed system as shown in Fig. 1 comprises two stages to: (i) evaluate the value of amplitude and total harmonic distortion (THD) of the captured wave using S-transform equations and (ii) classify the disturbance using Fuzzy-expert system according to the evaluated values.

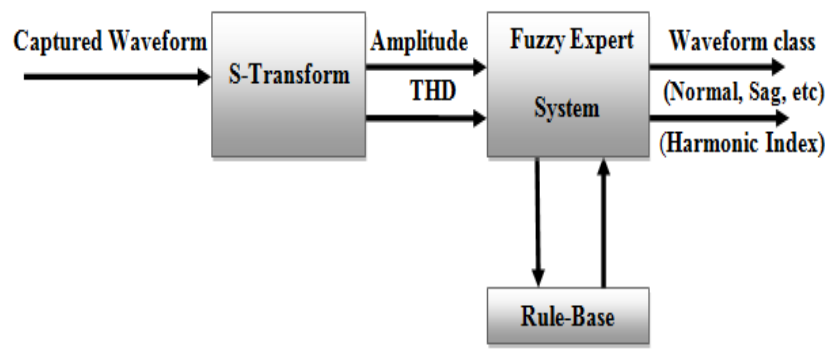

Figure 1. The block diagram of the proposed system

\subsection{The S-Transform}

Let $P[k T], k=0,1, \ldots \ldots, N-1$ denote a discrete time $P(t)$, with a time sampling interval of $T$. The discrete Fourier transform of the signal can be obtained as follows:

$$
P\left[\frac{n}{N T}\right]=\frac{1}{N} \sum_{k=0}^{N-1} P(k T) e^{-\left(\frac{i 2 \pi n k}{N}\right)}
$$

where, $n=0,1, \ldots ., N-1$ and the inverse discrete Fourier transform is:

$$
P[k T]=\frac{1}{N} \sum_{n=0}^{N-1} P(n / n T) e^{\left(\frac{i 2 \pi n k}{N}\right)}
$$

In the discrete case, the S-Transform is the projection of the vector defined by the time series $P(K T)$, onto a spanning set of vectors, the spanning vectors are not orthogonal and the elements of the S-Transform are not independent. Each basis vector (of the Fourier transform) is divided into localized vectors by an element- by- element product with the shifted Gaussians, such that the sum of these localized vectors is original basis vector. The S-Transform of a discrete time series $P(K T)$ is given by [15]:

$$
S\left[\frac{n}{N T}, j T\right]=\frac{1}{N} \sum_{m=0}^{N-1} P(m+n / n T) G(n, m) e^{\left(\frac{i 2 \pi m j}{N}\right)}
$$

where $G(n, m)=e^{-\left(2 \pi^{2} m^{2} / n^{2}\right)}=$ Gaussian function and $j, m, n=0,1, \ldots \ldots, N-1$.

The following steps are adapted for computing the discrete S-Transform:

1 Perform the discrete Fourier transform of the original time $P(K T)$ (with $\mathrm{N}$ points and sampling interval $T$ to get $P(m / n T)$ using the FFT routine. This is only done once.

2 Calculate the localizing Gaussian $G(n, m)$ for the required frequency $\left(\frac{n}{n T}\right)$.

3 Shift the spectrum $P(m / n T)$ to $P(m+n / n T)$ for the frequency $n / n T$ (one point addition)
4 Multiply $P(m+n / n T)$ by $G(n, m)$ to get $B\left(\frac{n}{n T}, \frac{m}{n T}\right)(\mathrm{N}$ multiplication).

5 Inverse Fourier transforms of $B\left(\frac{n}{n T}, \frac{m}{n T}\right) m / N T$ to $j$ to give the row of $S\left[\frac{n}{n T}, j T\right]$ corresponding to frequency $n / n T$.

6 Repeat steps 3, 4, and 5 until all the rows of $S\left[\frac{n}{n T}, j T\right]$ corresponding to all discrete frequencies $n / n T$ have been defined.

From Equation (3), it is seen that the output from the Stransform is a $(N \times M)$ matrix called the S-matrix whose rows pertain to frequency and columns to time. Each element of the S-matrix is complex valued. The choice of windowing function is not limited to the Gaussian function; other windowing functions are also implemented successfully.

\subsection{The Fuzzy Expert System}

Fuzzy logic $[16,17]$ refers to a logic system which represents knowledge and reasons in an imprecise or Fuzzy manner for reasoning under uncertainty. Unlike the classical logic systems, it aims at modeling the imprecise modes of reasoning that play an essential role in the human ability to infer an approximate answer to a question based on a store of knowledge that is inexact, incomplete, or not totally reliable. It is usually appropriate to use Fuzzy logic when a mathematical model of a process does not exist or does exist but is too difficult to encode and too complex to be evaluated fast enough for real time operation. The accuracy of the Fuzzy logic systems is based on the knowledge of human experts; hence, it is only as good as the validity of the rules. Fig. 2 shows the construction of Fuzzy expert system.

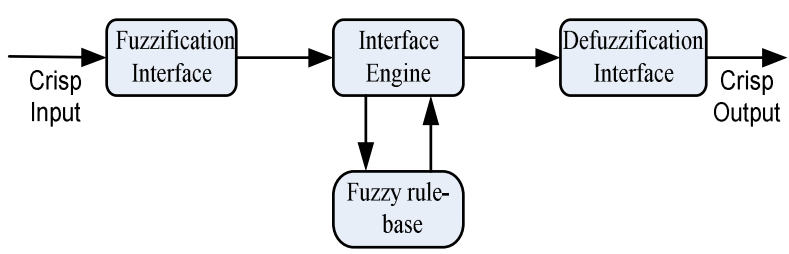

Figure 2. The construction of a Fuzzy expert system

\subsection{Implementation of the Proposed Methodology}

Many analysis results of PQ disturbances could be obtained from ST matrix, $S\left[\frac{n}{N T}, j T\right]$. The first extracted parameter is the amplitude 'A' which is the locus of maximum value of elements present in the column of the $\mathrm{S}$ matrix corresponding to the time. The total harmonic distortion (THD) is the second extracted parameter. It is calculated using the FFT routine, Equation (1) where,

$$
T H D=\frac{\sqrt{v_{2}^{2}+v_{3}^{2}+v_{4}^{2}+\cdots+v_{n}}}{v_{1}}
$$

Where, $v_{1}$ is the fundamental wave amplitude, $v_{2}, v_{3}, v_{4}, \ldots, v_{n}$ are the other frequencies (harmonic) order amplitudes.

For classifying the disturbance waveforms, five Fuzzy sets 
are chosen for the amplitude A, the first input of Fuzzyexpert, designated as VSA (very small amplitude), SA (small amplitude), NA (normal amplitude), LA (large amplitude), and VLA (very large amplitude). The total harmonic distortion (THD), the second input of Fuzzy-expert, has two Fuzzy sets that are designed as (Small value of THD) and (Large value of THD).

The input variables membership functions of the Fuzzy expert system are shown in Figs. 3 and 4.

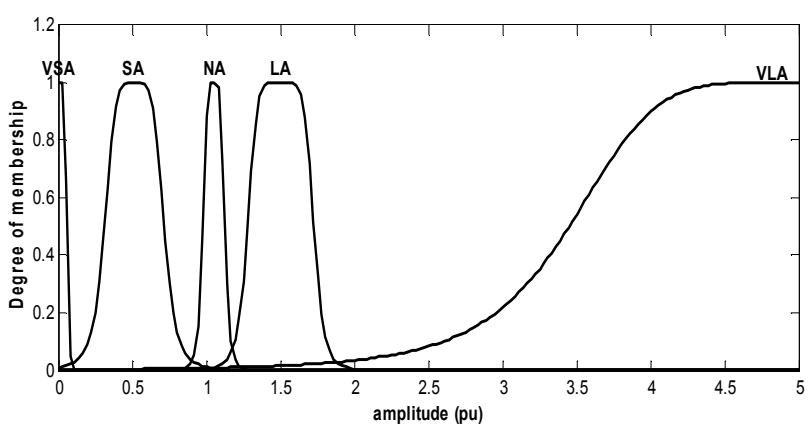

Figure 3. The First input membership function

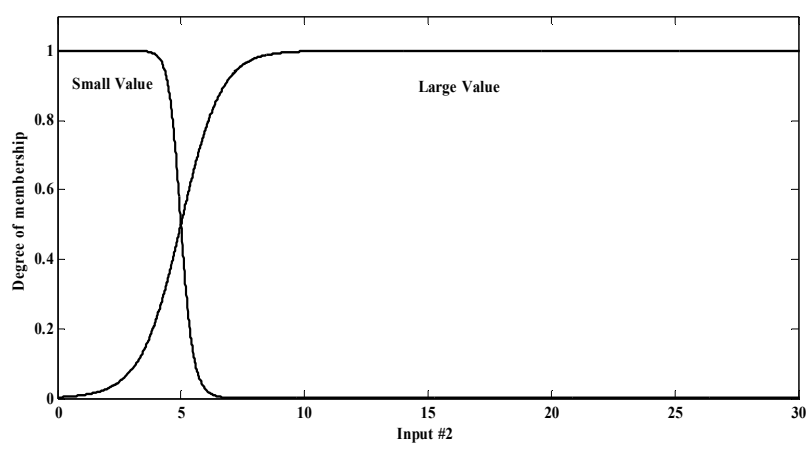

Figure 4. The second input membership function

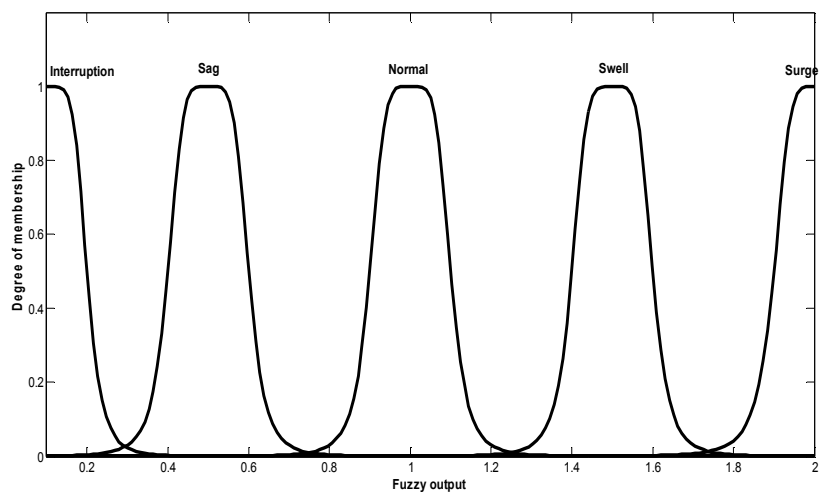

Figure 5. The first Fuzzy output membership function

The output membership function is defined by five sets. These sets are designated as interruption, sag, normal, swell, and surge.

The first output of the Mamdani Fuzzy system, Fig. 5, can assume values between 0 and 2 for the output, where,

Interruption $=0, \mathrm{Sag}=0.5$, Normal $=1$, Swell $=1.5$, Surge $=2$.

The second output of the Mamdani Fuzzy system, Fig. 6, can assume values between 0 and 1 for the output where, Pure wave $=0$, Distorted wave $=1$.

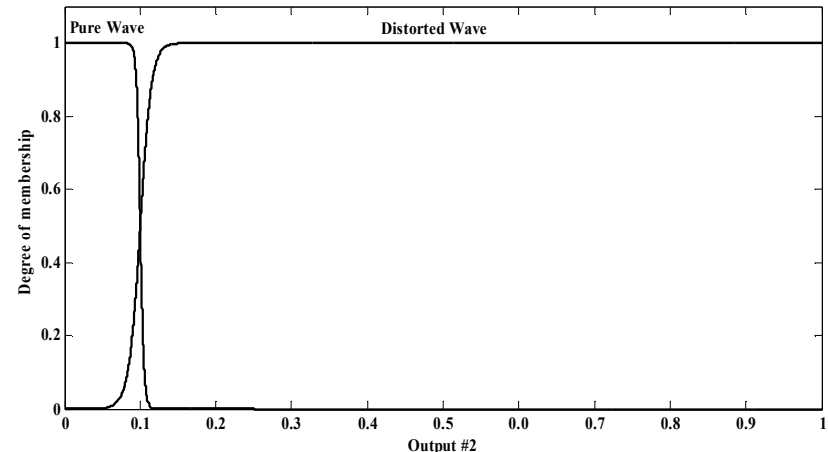

Figure 6. The second Fuzzy output membership function

The parameters of amplitude membership function are determined according to the definition of each PQ event.

The brief rule sets of Fuzzy expert system are below:

1 If Input \#1 is SA and the Input \#2 is Small value, Then the output \#1 is Sag, and the output \#2 is (pure wave)

2 If Input \#1 is VSA and the Input \#2 is Small value, Then output \#1 is Interruption, and output \#2 is (pure wave)

3 If Input \#1 is LA and Input \#2 is Small value, Then output \#1 is Swell, and output \#2 is (pure wave)

4 If Input \#1 is VLA and Input \#2 is Small value, Then output \#1 is Surge, and output \#2 is (pure wave)

5 If Input \#1 is NA and Input \#2 is Small value, Then output \#1 is Normal, and output \#2 is (pure wave)

6 If Input \#1 is NA and Input \#2 is Large value, Then output \#2 is (Distorted wave)

7 If Input \#1 is SA and Input \#2 is Large value, Then output \#1 is Sag, and output \#2 is (Distorted wave)

8 If Input \#1 is LA and Input \#2 is Large value, Then output \#1 is Swell, and output \#2 is (Distorted wave)

\section{Simulation Results}

In this study, power quality disturbances signals are seven signal disturbances including voltage sag, swell, interruption, surge, harmonic distortion, sag with harmonic and swell with harmonic.

The generated waveform has a frequency of $50 \mathrm{~Hz}$ and the voltage waveform sampled at a rate of $1.6 \mathrm{kHz}$, i.e. 32 samples per cycle. The general equation of generated wave form is:

$$
S(t)=A_{1} \sin (w t)+A_{2} \sin (3 w t)+A_{3} \sin (5 w t)
$$

where, $A_{1}$ is the amplitude of the fundamental wave and equal to 1 p.u, and $A_{2} \& A_{3}$ are the amplitudes of the third and fifth harmonic order imposed on the fundamental sine wave. The following case studies are presented to illustrate the aptness of the proposed system. 


\subsection{Normal Voltage}

The normal voltage is the rated operating voltage at rated frequency, also there is not any PQ disturbance on the fundamental wave. The general equation form of the generated wave is $S(t)=A_{1} \sin (w t)$, where, $A_{1}=1$ p.u and putting $A_{2}$, \& $A_{3}$ equal to zero as shown in Fig. 7-a. Fig 7-b shows the output of the $S$ transform by searching the maximum of each column of the $S$ matrix. Fig. 7-c shows the frequency contour of the captured wave using FFT analysis. The period of disturbance and the total output of the Fuzzy expert system are shown in the Figs. 8-a, 8-b and 8-c, respectively. From Fig 8-a, the magnitude equals one all over the period which is Normal. The second Fuzzy output equals zero, this means that the waveform does not contain harmonic distortion.
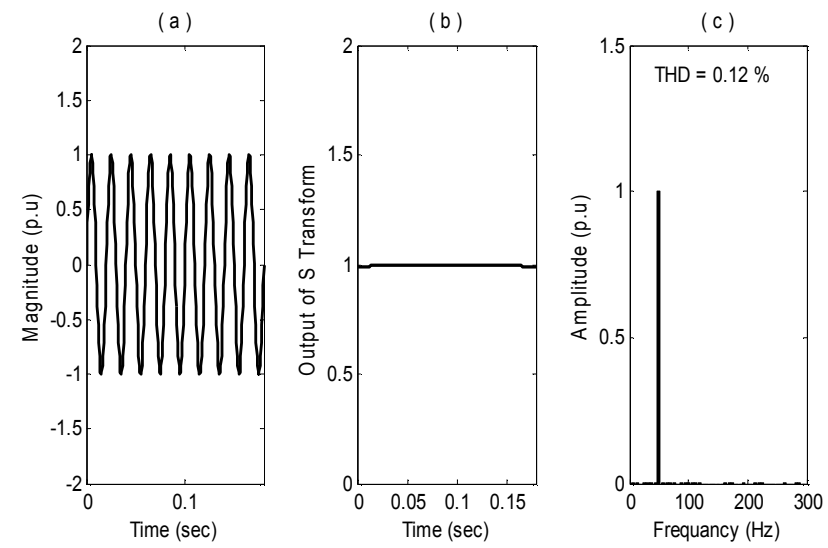

Figure 7. Normal voltage: (a) waveform, (b) magnitude time spectrum and (c) frequency contour
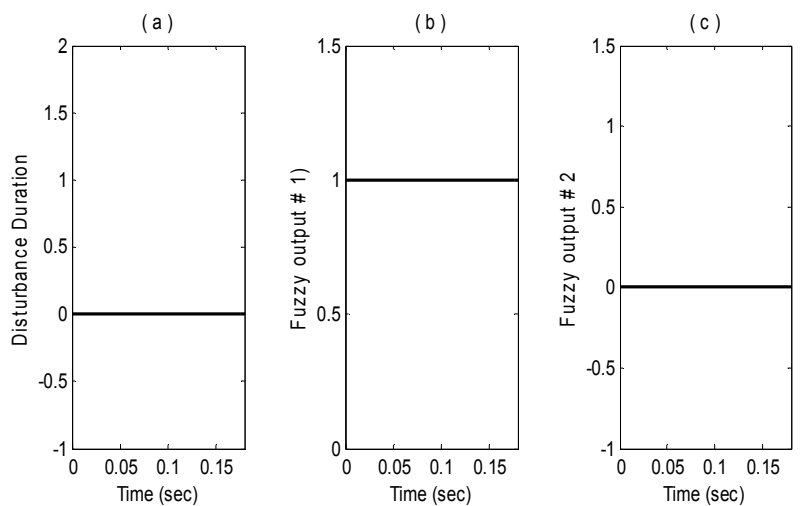

Figure 8. Normal voltage: (a) disturbance duration, (b) Fuzzy output \#1 (c) Fuzzy output \#2

\subsection{Voltage Sag}

Voltage sag is a decrease of $10-90 \%$ of the rated system voltage for duration of 0.5 cycles to $1 \mathrm{~min}$. The generated wave equation is $S(t)=A_{1} \sin (w t)$, where, $A_{1}=1$ p.u. and $A_{2}, \& A_{3}$ are equal to zero. By applying a decrease to the voltage magnitude to be 0.5 p.u for 3 cycles as shown in Fig. 9-a. Figs. 9-b and 9-c show the output of S transform and the frequency contour of the captured wave in order to calculate the total harmonic distortion. Fig. 10-a shows the period of disturbance while Fig. 10-b gives the classification of disturbance according to the amplitude. In this case the magnitude drop from one to 0.5 which means that the waveform contains sag. The second Fuzzy output equals zero, this means that the waveform does not contain harmonic distortion as shown from Fig. 10-c.
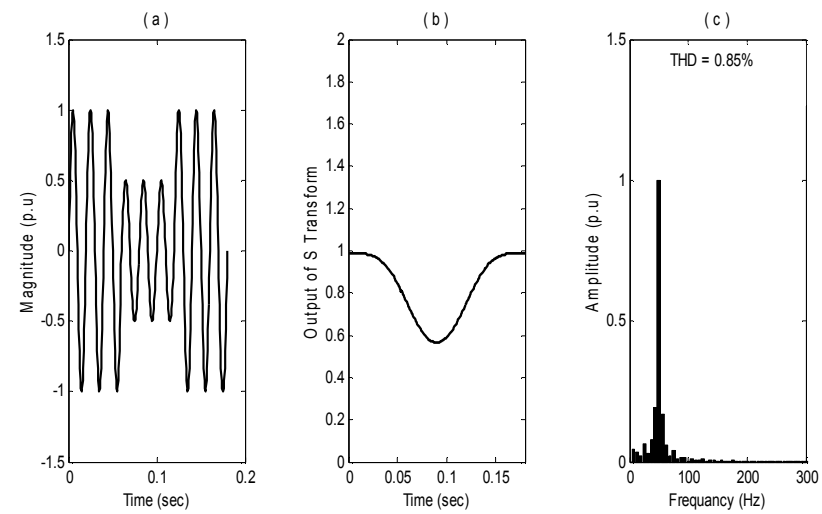

Figure 9. Voltage sag: (a) waveform, (b) magnitude time spectrum, (c) frequency spectrum
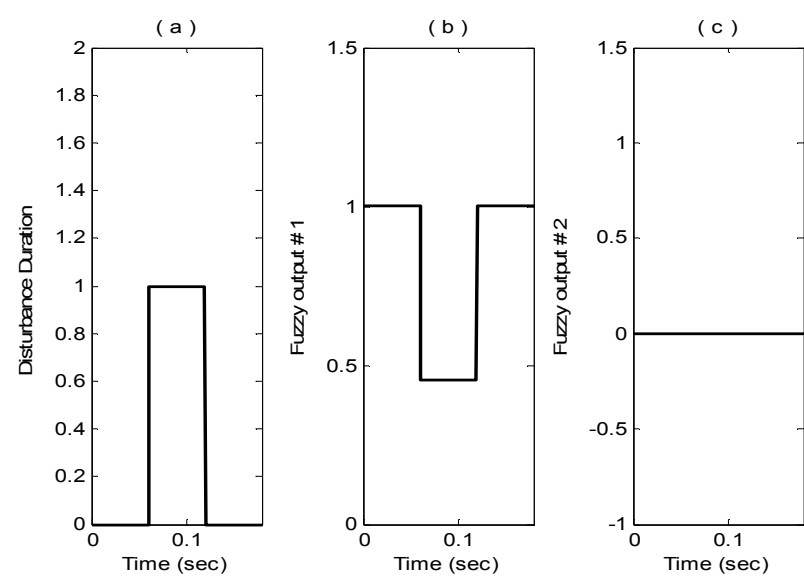

Figure 10. Voltage sag: (a) disturbance duration, (b) Fuzzy output \#1 (c) Fuzzy output \#2
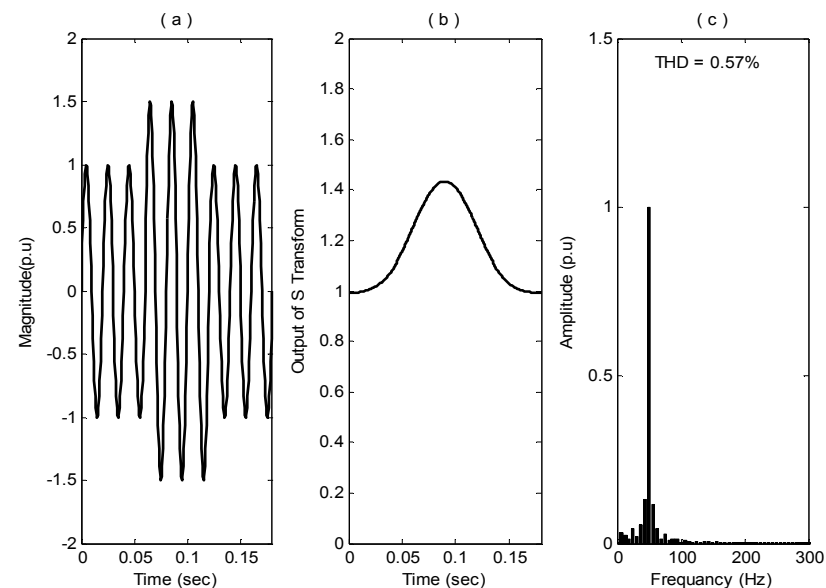

Figure 11. Voltage swell: (a) waveform, (b) magnitude time spectrum, (c) frequency spectrum 

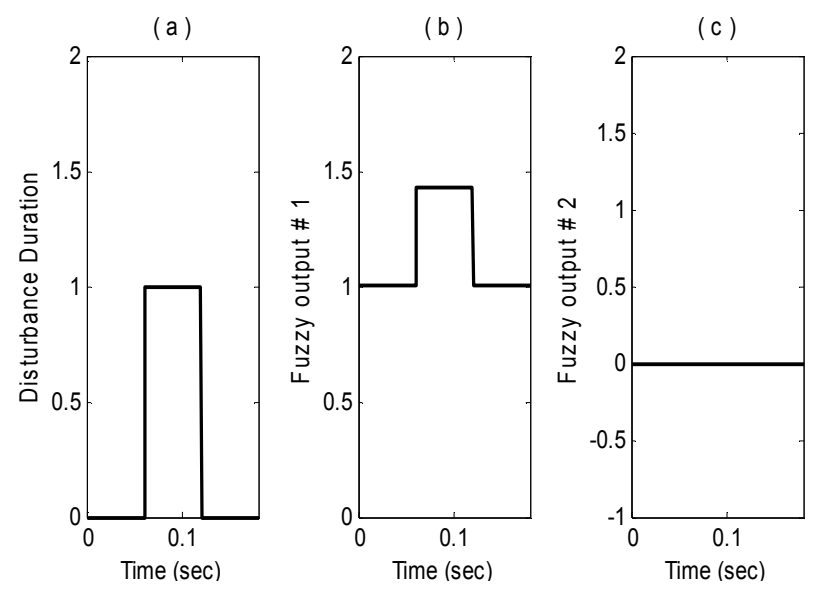

Figure 12. Voltage swell: (a) disturbance duration, (b) Fuzzy output \#1 (c) Fuzzy output \#2

\subsection{Voltage Swell}

In the case of voltage swell, there is a rise of 10 to $90 \%$ in the voltage magnitude for 0.5 cycles to $1 \mathrm{~min}$. the equation of the generated wave is $\mathrm{S}(\mathrm{t})=1 \sin (\mathrm{wt})$ and $\mathrm{A}_{2} \& A_{3}$ are equal to zero. Increasing of the voltage to $1.5 \mathrm{p}$.u for three cycles, the magnitude rises from one to $1.5 \mathrm{p} . \mathrm{u}$ as shown in Figs. 11-b and 12-b, this means that the waveform contains swell. The second Fuzzy output equals zero, this means that the waveform does not contain harmonic distortion.
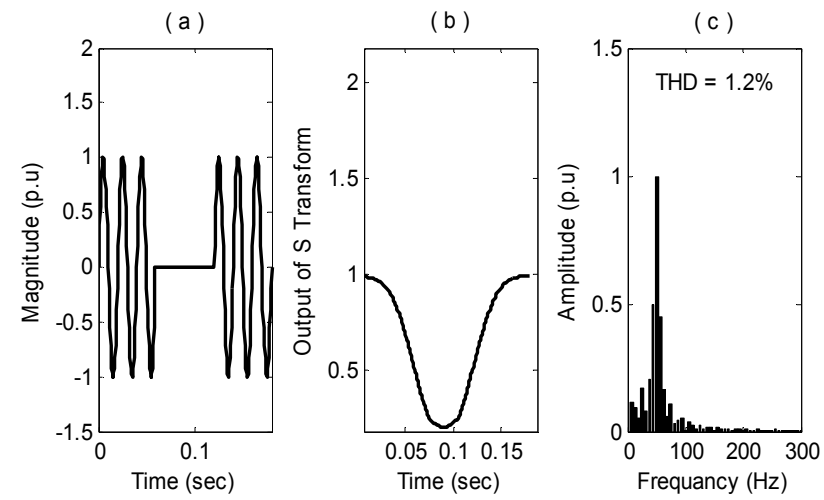

Figure 13. Voltage interruption: (a) waveform, (b) magnitude time spectrum and (c) frequency spectrum
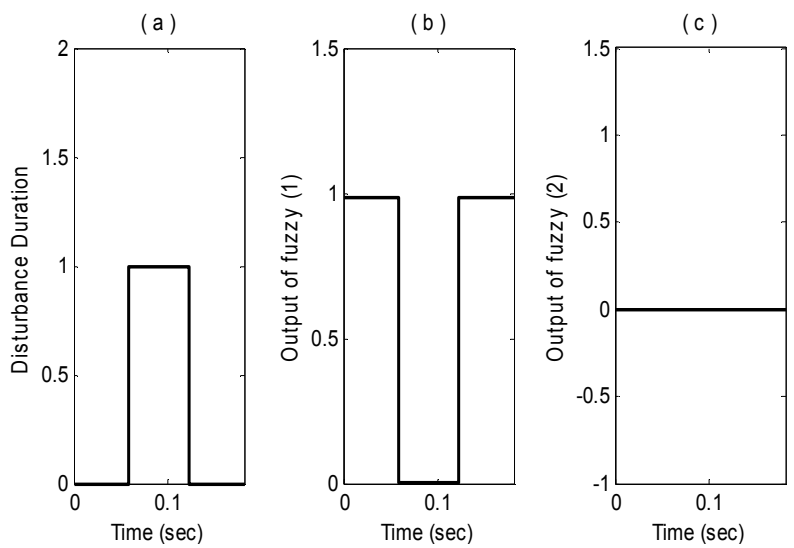

Figure 14. Voltage interruption: (a) Disturbance duration, (b) Fuzzy output $\# 1$ and (c) Fuzzy output \#2
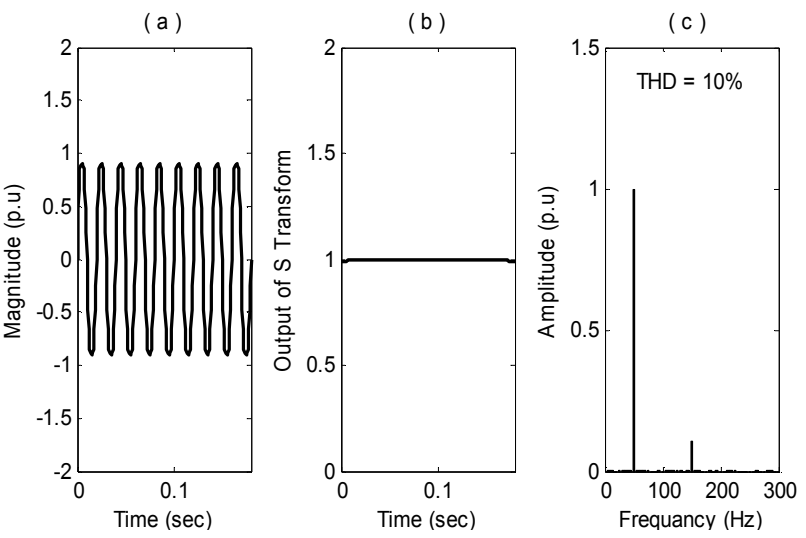

Figure 15. Voltage distortion: (a) waveform, (b) magnitude time spectrum and (c) frequency spectrum
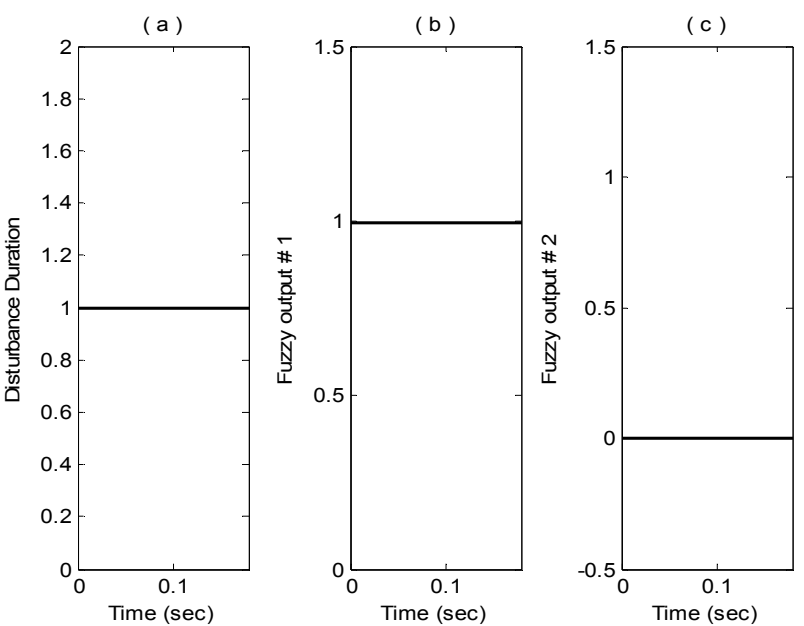

Figure 16. Voltage distortion: (a) disturbance duration, (b) Fuzzy output \#1 and (c) Fuzzy output \#2

\subsection{Voltage Interruption}

An interruption may be seen as a loss of voltage on a power system. Such disturbance describes a drop of $90-100 \%$ of the rated system voltage for duration of 0.5 cycles to $1 \mathrm{~min}$. The generated wave equation is $\mathrm{S}(\mathrm{t})=1 \sin (\mathrm{wt})$ with $\mathrm{A}_{2} \& A_{3}$ are equal to zero. By applying an interruption for three cycles as shown in Fig. 13-a, the magnitude drop from one to zero is shown in Figs. 13-b and 14-b. Fig. 14-c illustrates the second Fuzzy output, in this case the harmonic index of the captured wave is equal to zero. So, the wave is pure.

\subsection{Voltage Distortion}

Distortion of the voltage waveform occurs when the harmonic is generated. This is done by adding the third harmonic to the original sine wave so that the generated wave is represented by $\mathrm{S}(\mathrm{t})=1 \sin (\mathrm{wt})+\mathrm{A}_{2} \sin (3 \mathrm{wt})$ with $\mathrm{A}_{2}=0.105 \mathrm{p}$.u and the total harmonic distortion is $10 \%$. Fig. 15-a shows the waveform with a third harmonic for nine cycles while Fig. 16-c shows the second Fuzzy output, in this case the harmonic index of the captured wave is equal to one. Hence, the wave is distorted. 


\subsection{Sag with Harmonics}

The sag with harmonic disturbance is done by adding the third harmonic to a sag waveform so that it can be represented by the equation $S(t)=1 \sin (w t)+A_{2} \sin (3 w t)$ with $\mathrm{A}_{2}=0.105$ p.u. Fig 17 -a shows the generated waveform with a sag of 0.5 p.u and a third harmonic for the three cycles. Hence, the total harmonic distortion is $10 \%$. Fig. 18-b shows the first Fuzzy output, in this case, the amplitude is decreased from 1 to 0.5 p.u which means sag. The second Fuzzy output is shown in Fig. 18-c with harmonic index of the captured wave equals one so that the wave is distorted.
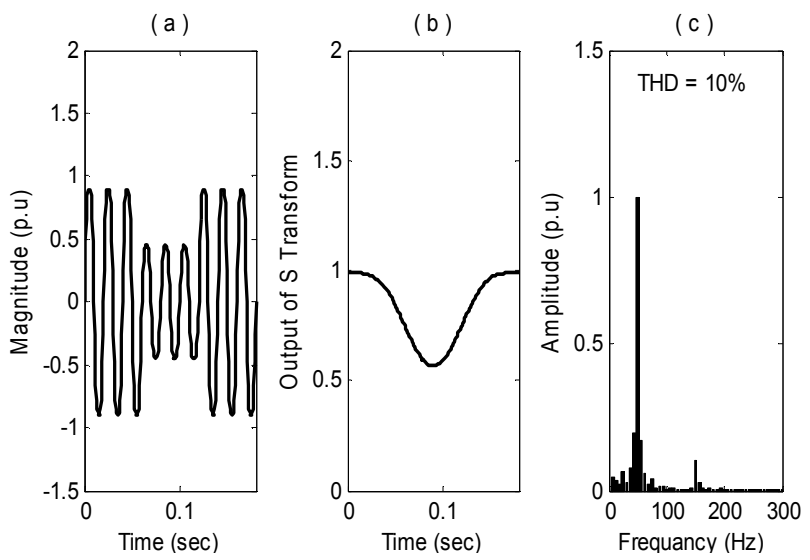

Figure 17. Sag with harmonic: (a) waveform, (b) magnitude time spectrum and (c) frequency spectrum
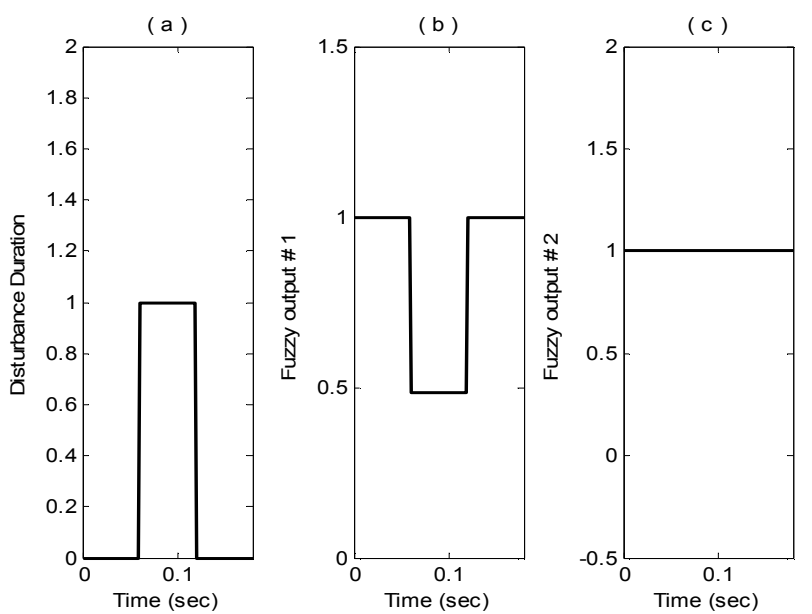

Figure 18. Sag with harmonic: (a) disturbance duration, (b) Fuzzy output \#1 and (c) Fuzzy output \#2

\subsection{Swell with Harmonic}

The swell with harmonic is done by adding the third harmonic to a sag wave and is represented by the equation $\mathrm{S}(\mathrm{t})=\mathrm{A}_{1} \sin (\mathrm{wt})+\mathrm{A}_{2} \sin (3 \mathrm{wt})$ with $\mathrm{A}_{1}=1$ p.u and $\mathrm{A}_{2}=0.105$ p.u. Applying swell of 1.5 p.u and a third harmonic for three cycles so that the total harmonic distortion is $10 \%$ as shown in Fig. 19-a. The first Fuzzy output shown in Fig. 19-b illustrates an increase of the amplitude of the waveform from 1 to $1.5 \mathrm{p}$.u which means swell. The second
Fuzzy output equals one, this means that the waveform contains harmonic distortion.
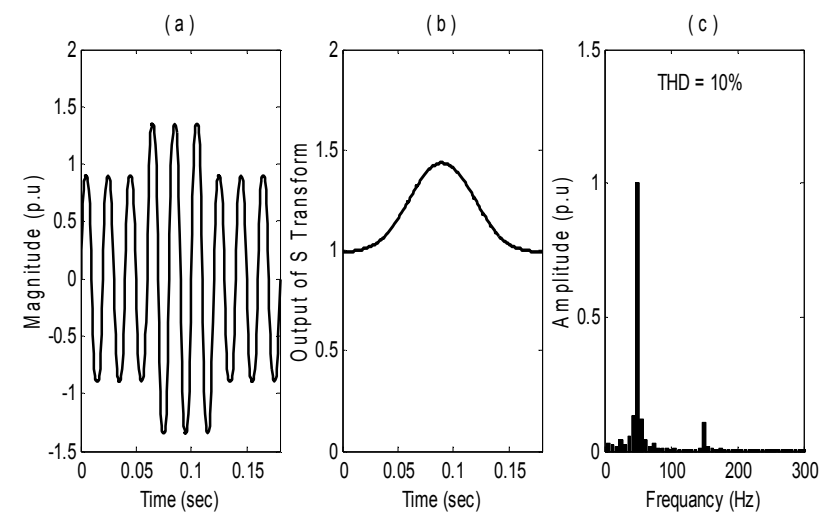

Figure 19. Swell with harmonic: (a) waveform, (b) magnitude time spectrum and (c) frequency spectrum
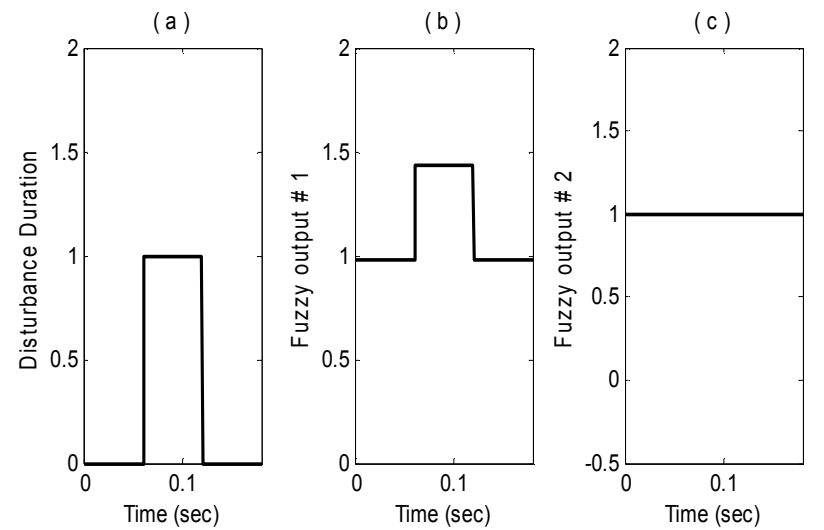

Figure 20. Swell with harmonic: (a) disturbance duration, (b) Fuzzy output \#1 and (c) Fuzzy output \#2
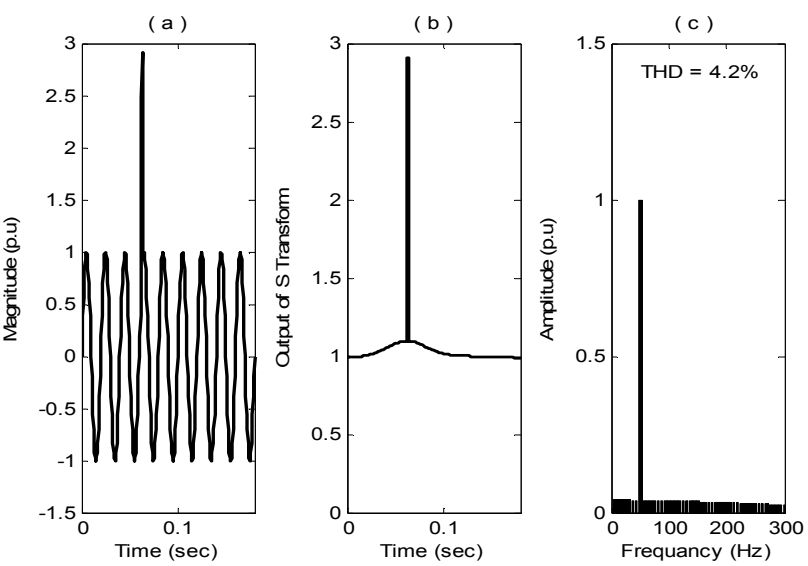

Figure 21. Voltage surge: (a) waveform, (b) magnitude time spectrum and (c) frequency spectrum

\subsection{Voltage Surge}

The surge occurs when the amplitude is suddenly increased from 1 to $3 \mathrm{p}$.u .for one-quarter cycle. In this case, the waveform is represented by $S(t)=A_{1} \sin (w t)+$ $A_{2} \sin (3 w t)$ with $A_{1}=1, A_{2}=0$ p.u. The amplitude is suddenly increased from 1 to 3 p.u as shown in Fig. 21-b. 
The first Fuzzy output shown in Fig. 22-b demonstrates a rises in amplitude to be (two) which indicate a case of surge. The second Fuzzy output equals zero, this means that the waveform does not contain harmonic distortion and the results are shown in Figs. 21 and 22.
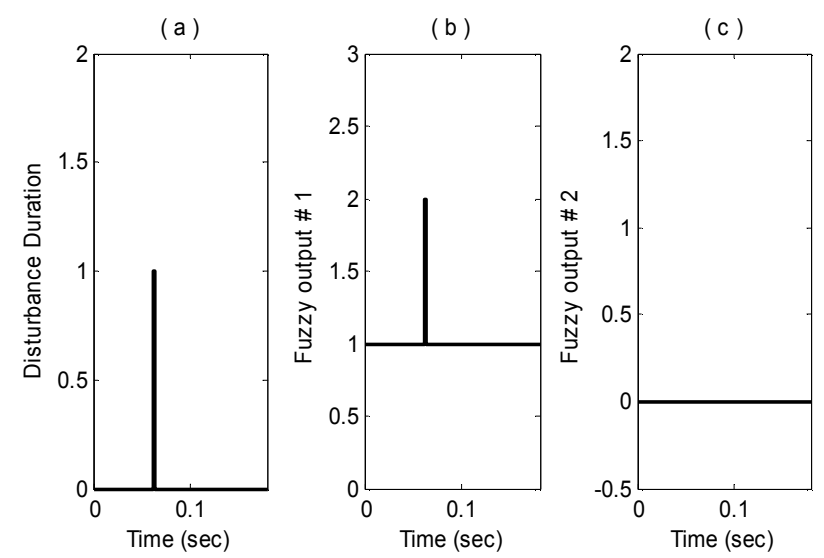

Figure 22. Voltage surge: (a) disturbance duration, (b) Fuzzy output \#1 and (c) Fuzzy output \#2

\section{Practical Data Results}

This section presents some of results obtained by applying the new approach on practical data. The practical data are obtained from the IEEE Project Group 1159.2 [19]. The sample frequency used is $\mathrm{F}_{\mathrm{S}}=15360 \mathrm{~Hz}$, or 256 samples per $60 \mathrm{~Hz}$ cycle.

\subsection{Case Study \#1}

Considering the captured waveform doesn't have any disturbance as shown in Fig 23-a. The Fuzzy first output is one which is normal and the second Fuzzy output equals zero, as shown in Fig. 24. This means that the waveform does not contain harmonic so that the wave is pure.
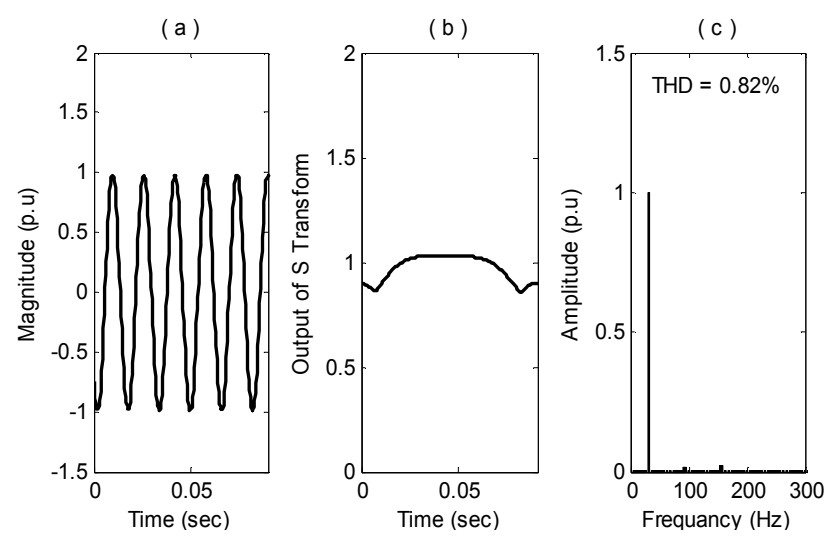

Figure 23. Case \#1: (a) waveform, (b) magnitude time spectrum and (c) frequency spectrum

\subsection{Case Study \#2}

In this case study, the sag in voltage waveform is detected using the $\mathrm{S}$ transform and is characterized using the results of
Fuzzy expert system. Fig. 25-a shows the voltage waveform with voltage sag. Fig. 26-b shows the output of the Fuzzy system which equals 0.5 . This means that the waveform contains sag. The output \#2 of Fuzzy expert system equals zero, this means that this waveform does not contain a harmonic distortion.
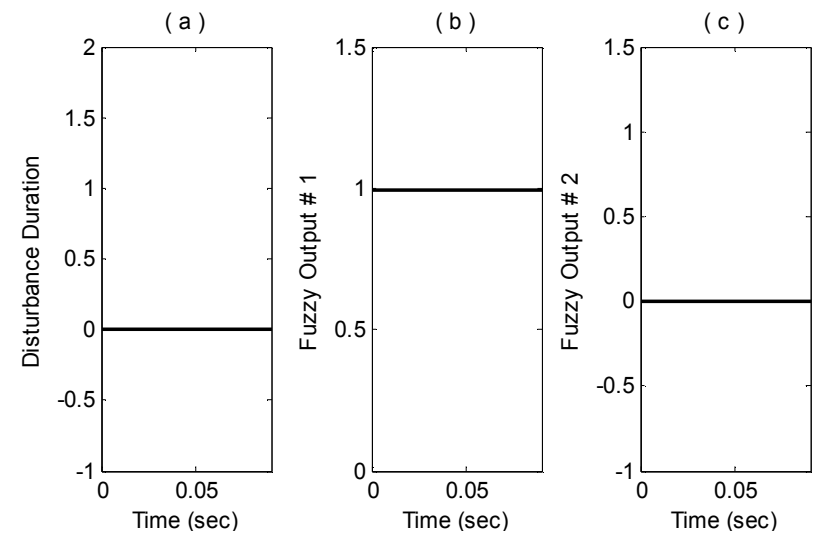

Figure 24. Case \#1: (a) disturbance duration, (b) Fuzzy output \#1 and (c) Fuzzy output \#2
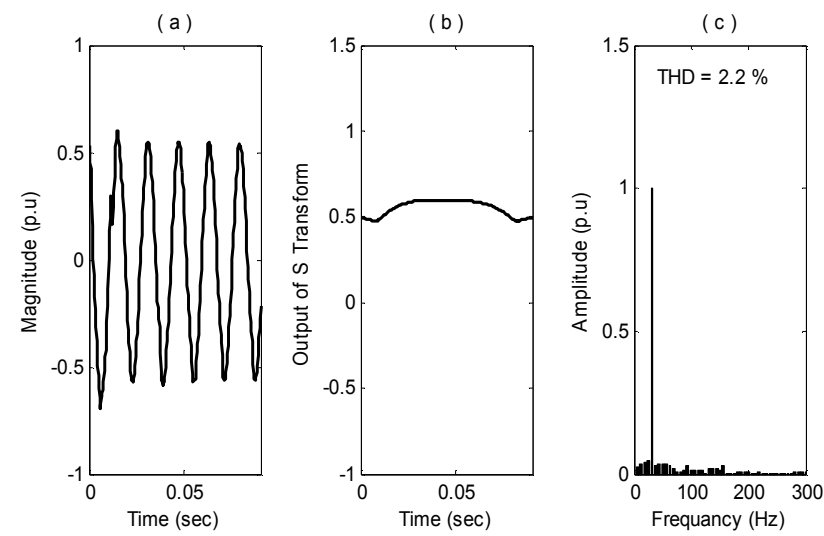

Figure 25. Case \#2: (a) waveform, (b) magnitude time spectrum and (c) frequency spectrum
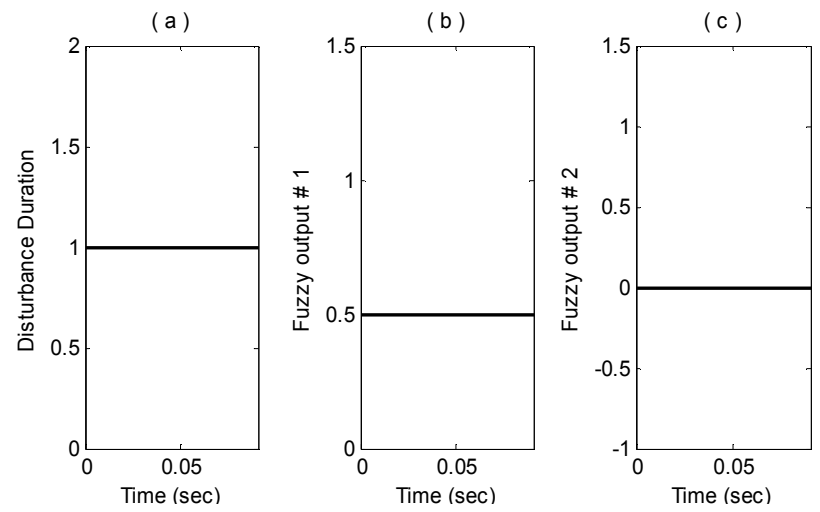

Figure 26. Case \#2: (a) disturbance duration, (b) Fuzzy output \#1 and (c) Fuzzy output \#2

\subsection{Case Study \#3}

The voltage waveform of this case is shown in Fig. 27-a. This waveform contains the sag power quality event with 
harmonics. As can be seen in Fig 28, the Fuzzy output clearly points the sag PQ event in the waveform, Fuzzy output \#1 is equal to 0.5 which refers to sag. The output \#2 of Fuzzy expert system equals one, this means that the waveform contains harmonic.
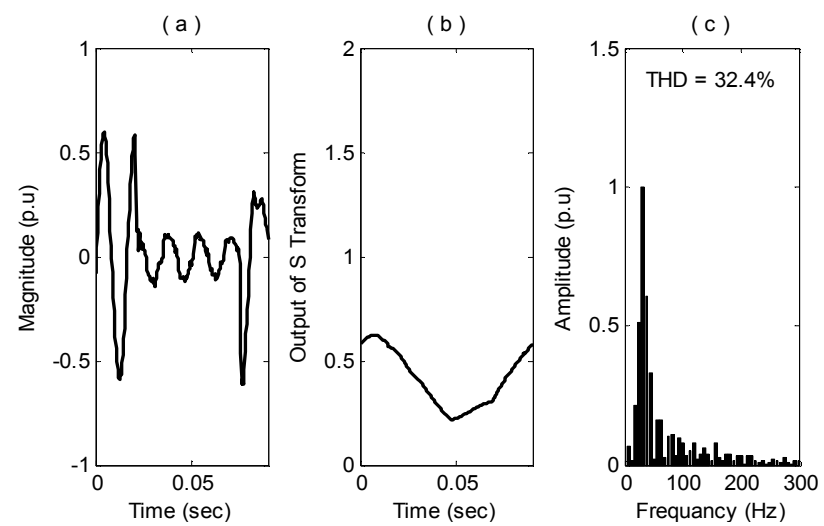

Figure 27. Case \#3: (a) waveform, (b) magnitude time spectrum and (c) frequency spectrum
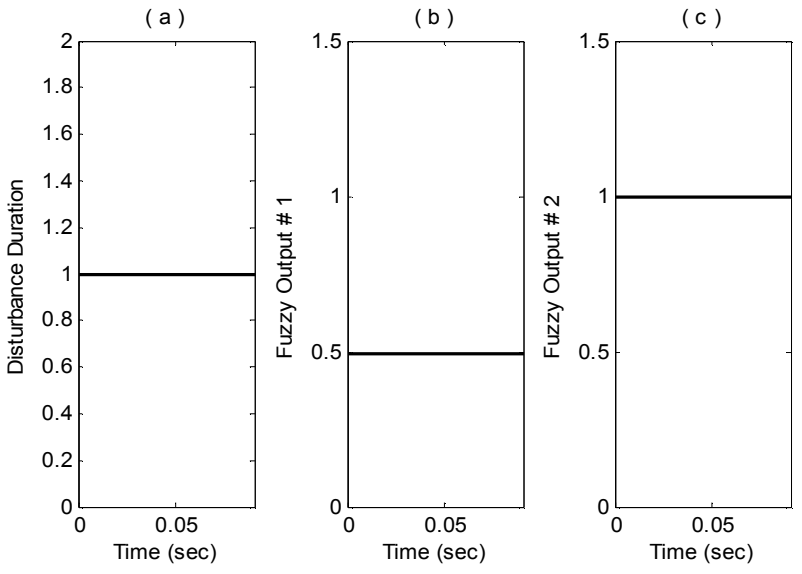

Figure 28. Case \#3: (a) disturbance duration, (b) Fuzzy output \#1 and (c) Fuzzy output \#2
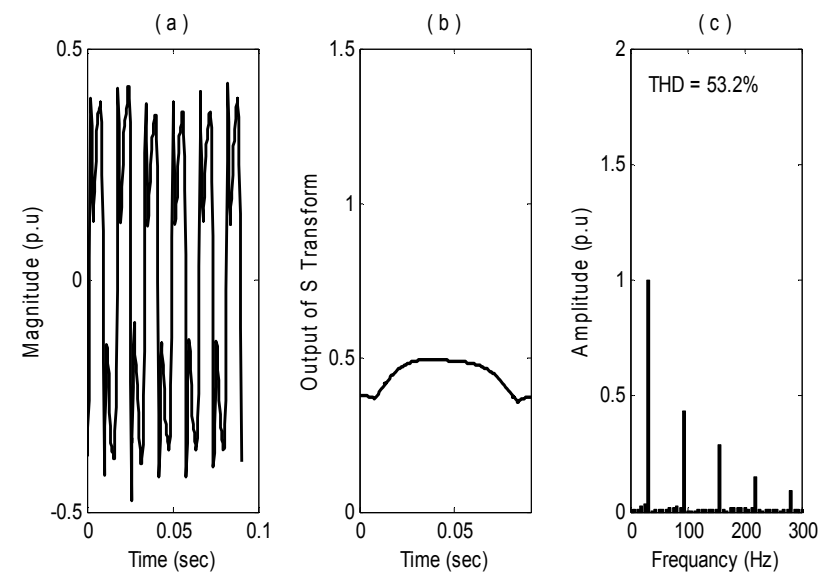

Figure 29. Case \#4: (a) waveform, (b) magnitude time spectrum and (c) frequency spectrum

\subsection{Case Study \#4}

In this case, the test waveform contains two power quality events; sag and harmonic distortion, with different harmonic order as shown in Fig 29-a. The output of S-transform is shown in Fig. 29-b. The duration of the disturbance is shown in Fig. 30-a. Fig. 30-b shows the first Fuzzy output which equals 0.5 . This means that the waveform contains sag. The second Fuzzy output equals one, i.e. the wave form is distorted.
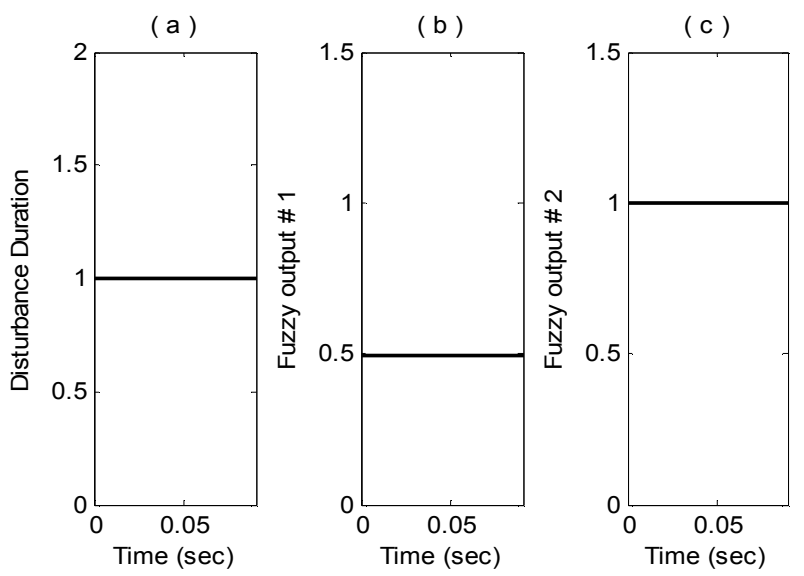

Figure 30. Case \#4: (a) disturbance duration, (b) Fuzzy output \#1 and (c) Fuzzy output \#2

\section{Comparison Between the Proposed Technique and Previous Published Works}

By comparing the performance of the proposed technique with those of other methods used for classification and identification of PQ disturbance such as the WT-based ANN method [6], WT and rule-based methodology [18], it is found that, there are a lot of parameters (more than 50 parameters) had to be determined to classify power disturbances in the previous mentioned methods. In this paper, using the STbased method, five types of single disturbance and two complex disturbances (sag with harmonic and swell with harmonics) can be classified using less number of calculated parameters which make the calculation period too short. On the other hand the wavelet transform coefficients at every scale belong to scope frequencies, not to a single frequency. Hence, it could not count the frequency amplitude accurately. ST has better frequency distinguish ability than WT. It is easily to identify the fundamental frequency of the of the disturbance signal of voltage sag, swell, and interruption and the other frequencies in case of harmonics providing a better visual disturbance degree comparing to DWT.

\section{Conclusions}

This paper presents a hybrid technique for characterizing PQ disturbances. The hybrid technique is based on Stransform for extracting two parameters, amplitude and harmonic indication from the captured distorted waveform. The two parameters are the inputs to Fuzzy-expert system 
that uses some rules on these inputs to characterize the PQ events in the captured waveform. The results show that the proposed hybrid technique has the ability to identify and classify the power system disturbances with high accuracy and small computation time comparing with other methods.

\section{References}

[1] IEEE Std 1159-1995, IEEE Recommended Practice for Monitoring Electric Power Quality, IEEE Inc., New York, pp.1-59, 1995.

[2] G. T. Heydt, P. S. Field, C. C. Liu, D. Pierce, L. Tu, G. Hensley, "Applications of the windowed FFT to electric power quality assessment", IEEE Trans Power Deliv., vol. 14, no. 4, pp. 1411-14166. 1999.

[3] Y. H. Gu, M. H. J. Bollen,'Time-frequency and time-scale domain analysis of voltage disturbances", IEEE Trans. Power Deliv., vol. 15, no. 4, pp. 1279-84, 2000.

[4] O. Poisson, P. Rioual, M. Meunier, "Detection and measurement of power quality disturbances using wavelet transform," IEEE Trans Power Deliv., vol. 15, no. 3, pp. 1039-1044, 2000.

[5] A. M. Gaouda, S. H. Kanoun, M. M. A. Salama, A. Y. Chikhani, "Pattern recognition applications for power system disturbance classification”, IEEE Trans. Power Deliv., vol. 17, no. 3, pp. 677-683, 2002.

[6] Z. L. Gaing, "Wavelet-based neural network for power disturbance recognition and classification," IEEE Trans. Power Deliv., vol. 19, no. 4, pp. 1560-1568, Oct. 2004.

[7] Abdelazeem A. Abdelsalama, Azza A .Eldesouky, and Abdelhay A. Sallam, "Classification of power system disturbances using linear Kalman filter and Fuzzy-expert system," Electr. Power Energy Syst., vol. 43, no. 1, pp. 688695, 2012.

[8] C. I. Chen, G. W. Chang, R. C. Hong, H. M. Li, "Extended real model of Kalman filter for time-varying harmonics estimation," IEEE Trans Power Deliv., vol. 25, no. 1, pp. 1726, 2010.
[9] X. Xiao, F. Xu, H. Yang, "Short duration disturbance classifying based on S transform maximum similarity", Int J Electr Power Energy Syst., vol. 31, no. 7, pp. 374-78, 2009.

[10] S. Suja, Suja Jovitha, "Pattern recognition of power signal disturbances using S Transform and TT Transform," Int J Electr Power Energy Syst., vol. 32, no. 1, pp. 37-53, 2010.

[11] P. K. Dash, B. K. Panigrahi, G. Panda. "Power quality analysis using s-transform," IEEE Transactions on Power Delivery, vol. 18 , no. 2, pp. 406-411, 2003

[12] S. Santoso, E. J. Powers, W.M. Grady, and A. C.Parsons, "Power quality disturbance waveform recognition using wavelet-based neural classifier -part 2: application," IEEE Trans. Power Delivery, vol. 15, pp 222-228, Jan. 2000.

[13] Y. Liao, J-B. Lee, "A Fuzzy expert system for classifying power quality disturbances," International Journal of Electrical Power and Energy Systems, vol. 26, no. 3, pp. 199205, 2004.

[14] W-M. Lin, C. Wu, C-H. Lin, F. S. Cheng, "Classification of multiple power quality disturbances using support vector machine and one-versus-one approach," International Conference on Power System Technology, vol. 2, pp. 1-8, 2006.

[15] R. G. Stockwell, L. Mansinha, and R. P. Lowe, "Localization of the complex spectrum: The S-transform," IEEE Trans. Signal Process., vol. 44, no. 4, pp. 998-1001, Apr. 1996.

[16] K.Passino, S.Yurkovich, Fuzzy Control, Longman: Addison Wesley, 1998.

[17] S. Guo, L. Peter, "A reconfigurable analog Fuzzy logic controller," Proceeding of the Third IEEE conference on IEEE World congress on computational intelligence, vol. 1, pp. 124128, June 1994.

[18] M. Kezunovic and L. Yuan, "A novel software implementation concept for power quality study," IEEE Trans. Power Del., vol. 17, no. 2, pp. 544-549, Apr. 2002.

[19] IEEE project group P1159.2. <http://www.standards.ieee.org>. 